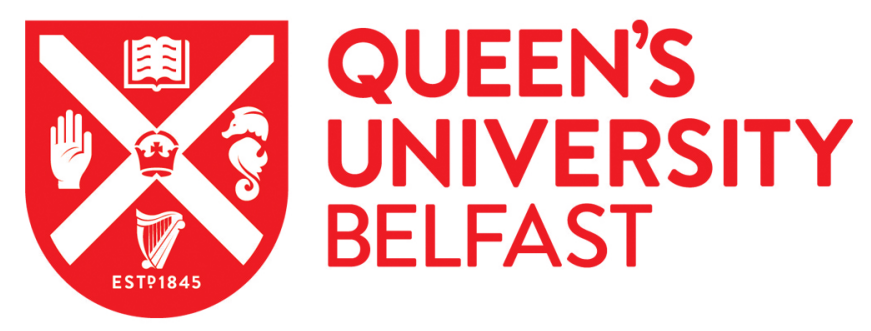

\title{
Dynamics of guided post-acceleration of protons in a laser-driven travelling-field accelerator
}

Hadjisolomou, P., Ahmed, H., Prasad, R., Cerchez, M., Brauckmann, S., Aurand, B., Schroer, A. M., Swantusch, M., Willi, O., Borghesi, M., \& Kar, S. (2020). Dynamics of guided post-acceleration of protons in a laser-driven travelling-field accelerator. Plasma Physics and Controlled Fusion, 62(11), [115023].

https://doi.org/10.1088/1361-6587/abb91a

Published in:

Plasma Physics and Controlled Fusion

Document Version:

Peer reviewed version

Queen's University Belfast - Research Portal:

Link to publication record in Queen's University Belfast Research Portal

Publisher rights

Copyright 2020 IOP. This work is made available online in accordance with the publisher's policies. Please refer to any applicable terms of use of the publisher.

\section{General rights}

Copyright for the publications made accessible via the Queen's University Belfast Research Portal is retained by the author(s) and / or other copyright owners and it is a condition of accessing these publications that users recognise and abide by the legal requirements associated with these rights.

Take down policy

The Research Portal is Queen's institutional repository that provides access to Queen's research output. Every effort has been made to ensure that content in the Research Portal does not infringe any person's rights, or applicable UK laws. If you discover content in the Research Portal that you believe breaches copyright or violates any law, please contact openaccess@qub.ac.uk. 


\title{
Dynamics of guided post-acceleration of protons in a laser-driven travelling-field accelerator
}

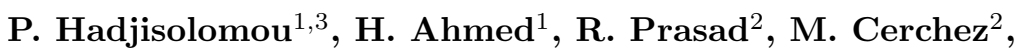 \\ S. Brauckmann ${ }^{2}$, B. Aurand ${ }^{2}$, A.M. Schroer ${ }^{2}$, \\ M. Swantusch ${ }^{2}$, O. Willi ${ }^{2}$, M. Borghesi ${ }^{1}$ and S. Kar ${ }^{1, *}$ \\ ${ }^{1}$ Centre for Plasma Physics, School of Mathematics and Physics, Queen's \\ University Belfast, Belfast BT7 1NN, United Kingdom \\ ${ }^{2}$ Institut für Laser-und Plasmaphysik, Heinrich-Heine-Universität, Düsseldorf, \\ Germany \\ ${ }^{3}$ Institute of Physics of the ASCR, ELI-Beamlines project, Na Slovance 2, \\ 18221 Prague, Czech Republic \\ E-mail: s.kar@qub.ac.uk
}

\begin{abstract}
By directing the laser-driven electromagnetic pulses along a helical path, one can achieve a travelling-field accelerator arrangement for simultaneous beam shaping and re-acceleration of laser-accelerated protons. The dynamics of guided acceleration of the transiting protons was studied by varying the length of the helical coil. Experimental data shows that the protons co-moving with the field region exhibit stronger focussing while increasing the coil length, with an increase of kinetic energy due to simultaneous post-acceleration. The net energy gain for a coil of constant pitch however saturates eventually when the post-accelerated protons overtakes the accelerating field region in due course. 3D particle tracing simulation underpins the dynamics of beam transport inside the coil, which highlights the requirement for a variable pitch coil geometry in order to sustain the post-acceleration over an extended coil.
\end{abstract}

Submitted to: Plasma Phys. Control. Fusion

\section{Introduction}

The laser-driven ion beams have been of increasing interest over the past decades due to a wide range of applications, which includes isotope production 1, hadron therapy [2, 3, 4, nuclear reactions studies [5], fast ignition [6] and studying ultra-fast dynamics of strong electric and magnetic fields during intense laser plasma interaction [7, 8, 9, 10]. The prospects associated with the laser-driven ion beams are highly promising, with a significant amount of research undergoing to improve further the quality of the accelerated ion beams.

So far the most robust and well understood mechanism of laser-driven accelerated ions is the Target Normal Sheath Acceleration (TNSA) 11. Over the past two decades there has been significant effort put forward, both experimentally [11, 12, 13, 14, 15] and theoretically [11, 16, 17, not only to explain the underlying physics, but also to improve the quality of the ion beams produced by this mechanism in light of the aforementioned applications. The ions (preferentially the protons, originally contained 
in the form of impurities on the target surface) in this mechanism are accelerated by a strong $(\mathrm{TV} / \mathrm{m})$ charge separation field at the rear surface of thin foils, which is created by the copious amount of hot electrons produced at the target front surface due to the interaction with intense laser [11.

One of the main advantages of a laser-driven source over the conventionally accelerated proton beams [11] is that the former provides a large number of particles in ultrashort bunches, an appealing feature for many ultra-fast applications, such as isochoric heating [15, 18, proton probing [10, radiolysis [19], radiobiology [20]. The proton energy spectrum obtained through TNSA mechanism follows an exponentially decaying profile [21]. Together with broad energy spectrum, the high divergence of the TNSA beams suits well for the proton-probing technique [9, 10], through which one can obtain snapshots of the field region with high temporal $(\sim \mathrm{ps})$ and spatial (10s of $\mu \mathrm{m}$ ) resolution. However, other potential applications, such as proton therapy for instance, primarily require a narrower energy spectrum and a collimated proton beam, which would allow targeted irradiation avoiding unnecessary damage to healthy tissues [2, 3, 4.

Various techniques have been proposed aiming on either improving the maximum energy, tailoring the spectrum or reducing the divergence of the proton beam. The most known of these experiments deploys permanent magnetic devices such as solenoid 22] or quadropole 23, laser-driven micro-lens [24, 25, 26, 27, 28], cylindrical lens 29. focusing and cascaded acceleration by double foil arrangement [30. On the other hand, techniques taking advantage of target engineering include the use of micro-structured [31, curved [32, 33, 34, hemisphere-cone [35] or flat-top cone [36] targets.

Recently a novel technique has been demonstrated [37. which allows simultaneous focusing, energy selection and post-acceleration of the laser driven ion beams. This technique is based on employing an ultra-short, high amplitude electromagnetic (EM) pulse (generated during the laser-foil interaction) guided along a helical coil target, which creates a strong electric field pattern that travels along the coil axis in synchronous with transiting protons of a narrow energy band, enabling guided post-accelration (at a rate of the order of $\mathrm{MeV} / \mathrm{mm}$ ) of the proton bunch over an extended $\sim \mathrm{cm}$ length. Deploying this technique at the ARCTURUS Laser of $200 \mathrm{TW}$, protons of $\sim 5 \mathrm{MeV}$ were simultaneously collimated and post accelerated to $\sim 10 \mathrm{MeV}$, as discussed in ref. 37.

In order to further optimise the performance of the coil targets, the effect of increasing the coil length is studied in this paper. While increase in the maximum proton energy is expected with increase in the length of the coil, the accelerated bunch of protons gradually overrun the accelerating field region in case of an extended coil of given pitch and radius, as illustrated by the experimental data presented in this paper. Particle tracing simulations corroborate the experimental findings and helped designing variable pitch helical coils to mitigate the premature energy saturation effect in an extended coil.

\section{Experimental Setup}

The experiment was conducted employing the ARCTURUS $200 \mathrm{TW}$ Ti:sapphire laser system located at the Institute of Laser and Plasma Physics of the University of Dusseldorf [38, 39]. The laser delivered short pulses in $\sim 30 \mathrm{fs}$ pulse duration, containing $\sim 3.0 \mathrm{~J}$ of energy after the compression. The laser beam was focused by an $\mathrm{f} / 2$ off-axis parabola to a spot of $\sim 4 \mu \mathrm{m}$ (FWHM), providing a peak intensity on the 


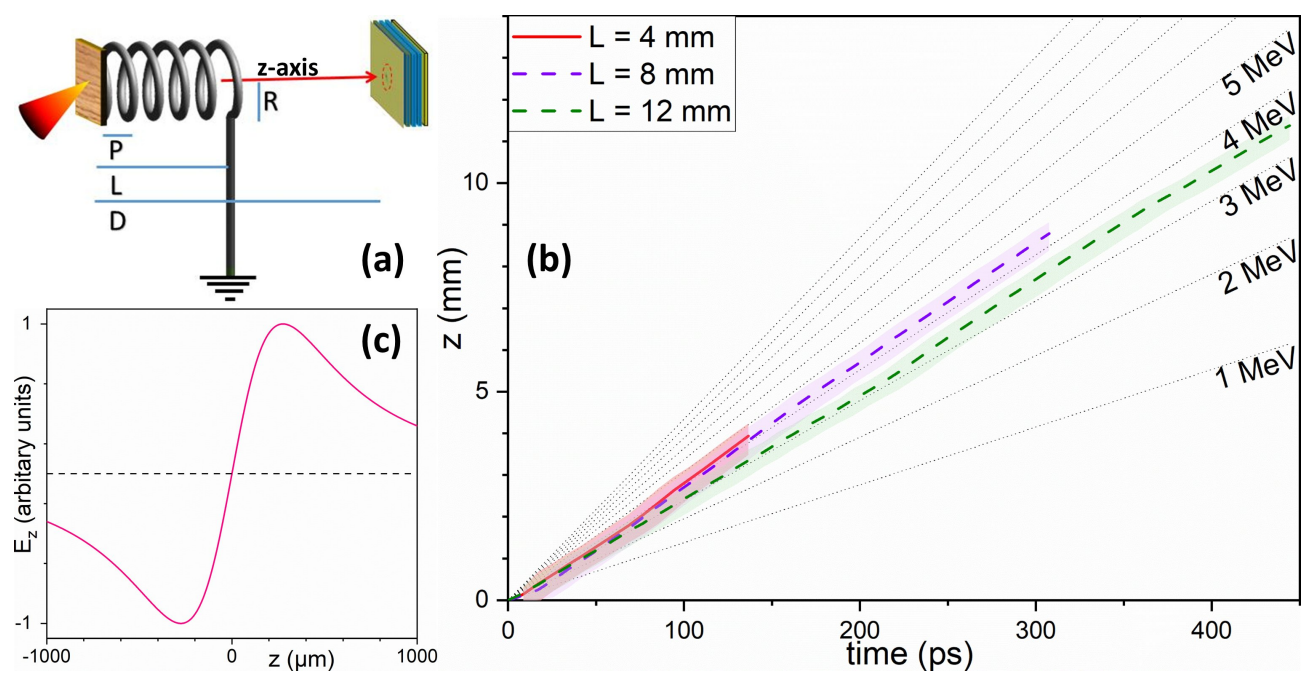

Figure 1. (a) A schematic of the experimental setup is shown. The cone indicates the laser beam interacting with the $\mathrm{Al}$ foil attached to the front side of the coil target, while the red arrow indicates the protons arriving at the detector after travelling through the coil. $R, P$ and $L$ represent the coil radius, pitch and length respectively, while $D$ is the foil to RCF distance. (b) The position-time graphs along the coil axis of the field region produced by the EM pulse are shown for the three coils of different lengths, $\mathrm{L}$ (coil-1 of $L \sim 4 \mathrm{~mm}$ (red), coil-2 of $L \sim 8.5 \mathrm{~mm}$ (purple) and coil-3 of $L \sim 11.5 \mathrm{~mm}$ (green), shown in Fig. 2 The shaded region for each line represents the FWHM extent of the field region inside the coil due to the profile of the EM pulse. The position-time graphs of the protons of various energies (as indicated on the right side of the graph) are over-plotted to show the range of energies of protons co-moving with the electric field region. (c) The plot shows the position of the longitudinal component of the electric field along the coil axis as a function of propagation time, where the peak of the EM pulse is located on the solid/dashed lines, while the FWHM of the pulse is represented by the shaded area.

target of $\sim 2 \times 10^{20} \mathrm{Wcm}^{-2}$.

The targets consisted of coils attached to the rear side of $6 \mu \mathrm{m}$ thick aluminium (Al) foils, as shown in Fig. 1(a). All coils were made of $80 \mu \mathrm{m}$ diameter Al wire, with an internal radius of $350 \mu \mathrm{m}$, and an average coil pitch, $P$, of $\sim 230 \mu \mathrm{m}$. The proton beam generated at the rear surface of the Al foil travelled through the coil, while the rear of the coil was connected to the ground. Three targets were examined, named as "coil-1", "coil-2" and "coil-3", with coil lengths, $L$, of $3.93 \mathrm{~mm}, 8.55 \mathrm{~mm}$ and $11.38 \mathrm{~mm}$ respectively.

Radio-Chromic Film (RCF) [40] stacks were placed at $\sim 33 \mathrm{~mm}$ from the foil of the coil target to measure the spatial and spectral profiles of the proton beam. A combination of calibrated HDV2 and HD810 types of Gafchromic 40 RCFs layers was used in the stacks.

\section{Results}

While irradiating $6 \mu \mathrm{m}$ thick $\mathrm{Al}$ foils (without a coil attached to its rear surface) with the laser pulse, proton beams of energy up to $\sim 7 \mathrm{MeV}$ with a temperature of $\sim 1 \mathrm{MeV}$ were typically produced, similar to that shown in ref. 37]. The beam spatial and 
Dynamics of guided post-acceleration of protons in a laser-driven travelling-field accelerator4
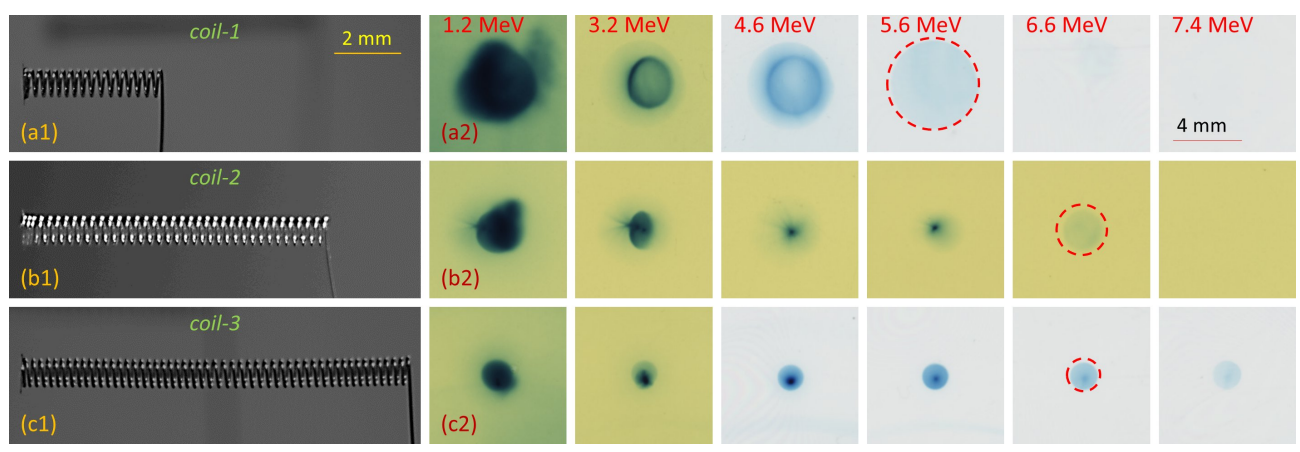

Figure 2. Images of helical coil targets of length (a1) coil-1 of $\sim 4 \mathrm{~mm}$ (b1) coil-2 of $\sim 8.5 \mathrm{~mm}$ (c1) coil-3 of $\sim 11.5 \mathrm{~mm}$. All three targets were made of $80 \mu \mathrm{m}$ diameter $\mathrm{Al}$ wire with an internal coil radius of $350 \mu \mathrm{m}$, average coil pitch of $230 \mu \mathrm{m}$ and $6 \mu \mathrm{m} \mathrm{Al}$ foils attached on the coils' front entrances. (a2), (b2), (c2) are the corresponding raw RCF data obtained in the experiment. The red dashed circle represents the geometrical projection of the coil's exit aperture on the RCF plane and the labels at the top of the RCF images correspond to their Bragg peak proton energies.

spectral profiles were similar to those typically produced by the TNSA mechanism, viz. a broad exponential spectrum and full-angle divergence of $\sim 30^{\circ}$. Using this as a reference, coil targets of different lengths were deployed in order to study the effect of the coil length on the transiting protons. As shown in Fig. 2(a2), obtained by using the short coil of $\sim 4 \mathrm{~mm}$ length (coil-1), a pronounced ring pattern in the beam profile of low energy protons was observed, which is significantly smaller than the geometrical projection of the coil exit aperture on the RCF plane (marked by a red dashed circle on the RCF layer corresponding to the Bragg peak proton energy of $5.6 \mathrm{MeV}$ ). The ring pattern corresponds to a piling of protons being deflected inwards by the EM pulse [9, 10, 37] travelling along the wire of the coil. The ring pattern appears to be smallest in diameter, i.e. the focusing effect appears to be strongest, for the proton energy of $\sim 3.2 \mathrm{MeV}$. This indicates that the beam is partially focused in this case, most likely due to the short length of the coil, however suggesting the existence of a strong focusing field inside the coil.

The formation of the ring features of varying diameters for different proton energies is due to the synchronisation between the protons travelling along the coil axis and the EM pulse travelling along the coil wire. As can be seen in Fig. 1(b), the EM pulse remains synchronised in the case of the coil-1 with $\sim 4 \mathrm{MeV}$ protons, albeit for a limited time due to the short length of the coil $(\sim 4 \mathrm{~mm})$, resulting in a partially focused beam appearing as a ring in the experimental data. The EM pulse is expected to have an insignificant effect on the protons of energy either side of the synchronisation window (i.e. the range of proton $\sim(3-5) \mathrm{MeV}$ for the coil-1, as shown in Fig. 1(b)). Therefore, the protons of energies above $5 \mathrm{MeV}$ (for instance, see the RCF layer corresponding to proton energy of $5.6 \mathrm{MeV}$ in Fig. 2(a2)) show a geometrically apertured beam footprint by the coil without any ring feature.

The focusing effect is expected to become stronger as the length of the coil is increased, while keeping the same radius and pitch of the coil. As shown in Fig. 1(b), the coil-2 has the same synchronisation window as the coil-1. However, the synchronised bunch of protons spends a significantly longer time inside the field 
region due to the extended coil length. This results, as shown in Fig.2(b2), in a tightly focused beam of protons at the centre of the coil's geometrical projection, compared to the ring patterns observed in the case of coil-1 (Fig. 2(a2)). The protons co-moving with the field region also experience the longitudinal electric field along the coil axis, resulting the guided bunch of protons being accelerated to higher energies. This can be clearly seen in Fig. 2(b2), where the focused beam of protons was observed in the RCF layer corresponding to $5.6 \mathrm{MeV}$.

As can be seen in Fig. 1.(b), the $5.6 \mathrm{MeV}$ protons originating from the foil travel much faster than the field region inside the coil, hence would arrive at the RCF layer unperturbedly. Analysis of the proton beam dose profile for the layer corresponding to 5.6 MeV proton in Fig. 2(a2) in fact reveals superimposition of two distinct Gaussian populations as shown in Fig. 3(a); a tightly focused central spot which is produced by the $(3-5) \mathrm{MeV}$ protons from the foil being simultaneously focussed and accelerated to $5.6 \mathrm{MeV}$, and a wider footprint which corresponds to the $5.6 \mathrm{MeV}$ protons from the foil being geometrically apertured by the coil. The guided post-acceleration by the coil-2 producing the former beam profile (the tightly focussed beam at $5.6 \mathrm{MeV}$ protons) can also be seen in the on-axis proton spectrum obtained from the RCF data, as shown in Fig. 3. (b). Compared to the typical proton spectrum obtained from a flat foil, the coil-2 produced a pronounced spectral peak centred aroun $5.6 \mathrm{MeV}$, with a significantly higher flux than obtained from the flat foil targets at similar proton energies.

The dual beam feature is even more pronounced in the data shown in Fig. 2(c2) obtained from coil-3. However, despite of a significant coil length, allowing protons to experience the accelerating field for significantly longer time, a focused beam up to $6 \mathrm{MeV}$, i.e. similar to what produced by the coil-2, was produced. As shown in Fig. 1 (b), the coil-3 had a narrow synchronisation window around $4 \mathrm{MeV}$ across the entire coil length. Therefore, it is conceivable that the protons after a few $\mathrm{MeV}$ of post-acceleration travel sufficiently ahead of the field region to attain any further energy gain. This is a major limitation for the helical targets, which in principle can be addressed by either decreasing the radius or increasing the pitch gradually along the coil axis.

\section{Simulations}

In order to corroborate our understanding of the dynamics of post-acceleration inside the coil target, as discussed above referring to the experimental data, particle tracing simulation employing PTRACE code [41] were carried out. In the simulations, coil geometries similar to those used in the experiment were defined and an EM pulse with a Gaussian half width half maximum rising of $5 \mathrm{ps}$ and decaying of $10 \mathrm{ps}$ was set to travel along the wire with a speed of $0.96 c$, where $c$ is the speed of light. These EM pulse parameters were taken from the ref. 37, which were obtained by proton probing measurements under similar laser and target parameters. The protons were set to originate from the centre of the entrance aperture of the coil, mimicking the spectrum and divergence of the beam obtained from the reference flat foil shots taken in the experiment. Fig. 4 shows the PTRACE simulations corresponding to the experimental conditions shown in Fig. 2 obtained from the three coils of different lengths. As can be seen, the simulations show an excellent agreement with the experiment, both in terms of the spatial profile and the energy of the guided proton beam. For instance, the ring-features in case of coil-1 are clearly visible in Fig. 4(a2), which are of similar sizes 
Dynamics of guided post-acceleration of protons in a laser-driven travelling-field accelerator6
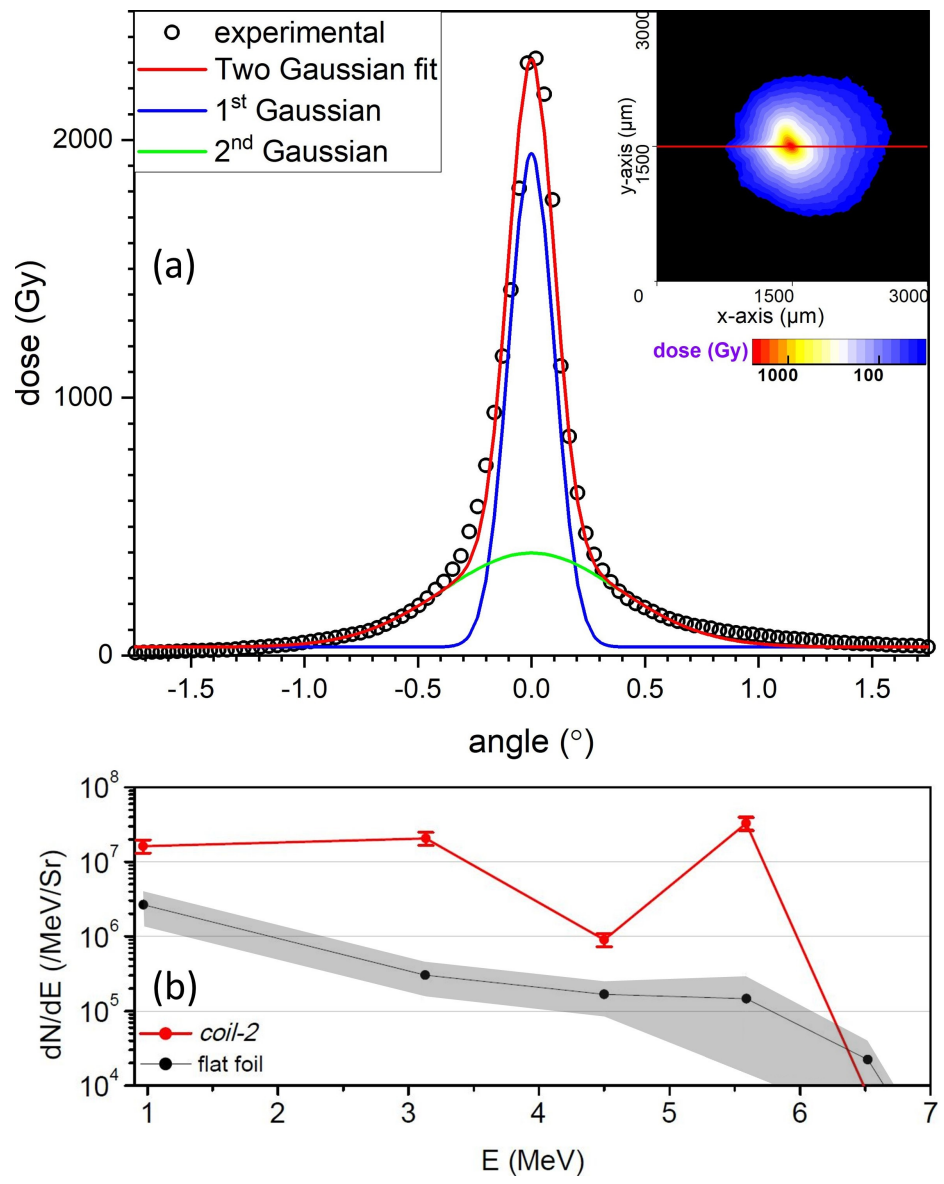

Figure 3. (a) Dose profile across the RCF showing two distinct Gaussian populations. The data shown in the insert correspond to the fourth RCF layer in Fig. 2 b2) obtained for the coil-2 case. The dose line-out across the data, shown by the red horizontal line in the insert, is fitted with two Gaussian distributions, one with FWHM $\approx 525 \mu \mathrm{m}$ (background population) and the other of $\mathrm{FWHM} \approx 150 \mu \mathrm{m}$, which represents the focused population of protons with a divergence full angle of $\sim 0.3^{\circ}$. (b) The red line shows the on-axis proton spectrum produced by the coil-2 considering the area of the central spot ( $\sim 200 \mu \mathrm{m}$ diameter). The shaded grey region shows the shot-to-shot variation (from estimated three shots with $6 \mu \mathrm{m} \mathrm{Al}$ foil targets taken during the experiment) in proton spectrum produced by flat foil targets, while considering $\sim 200 \mu \mathrm{m}$ diameter spot on the RCF along the beam axis.

as the respective RCF layers shown in Fig. 2(a2). Furthermore, the tightly focused beams observed in the experiment for the coil- 2 and coil-3 have been reproduced quite well by the simulations. The best match with the experimental data in all cases is obtained for an EM pulse peak amplitude of $3.2 \pm 0.3 \mu \mathrm{C} / \mathrm{m}$, which is similar to that measured for the interaction condition, as reported in ref. 37.

The simulations shown in Fig. 4 also indicate no significant increase in guided beam's energy by increasing the coil length from $8 \mathrm{~mm}$ to $12 \mathrm{~mm}$, which is in-line with the experimental data. The effect of the coil length for a constant pitch helical coil was 
Dynamics of guided post-acceleration of protons in a laser-driven travelling-field accelerator 7

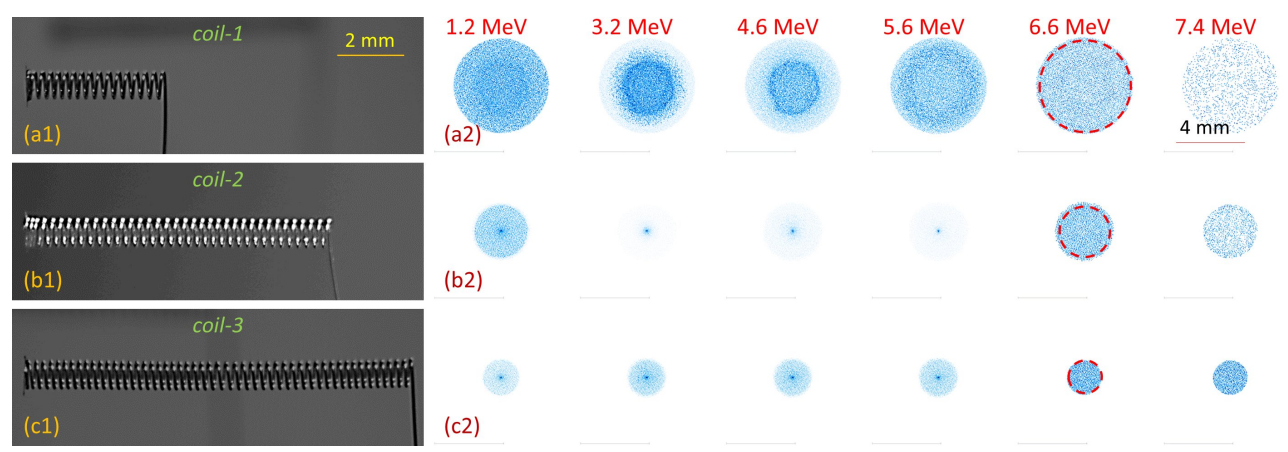

Figure 4. Images of the three helical coil targets shown in Fig. 2 (a1) coil1 (b1) coil-2 (c1) coil-3. (a2), (b2), (c2) are the simulated RCF images for the respective coil targets produced by the PTRACE simulations. The simulated $\mathrm{RCF}$ images are of $8 \times 8 \mathrm{~mm}$ size and the red circle corresponds to the geometrical projection of the coil apertures on the RCF plane. The labels at the top of the $\mathrm{RCF}$ images correspond to their Bragg peak proton energies.

further investigated through simulations. For instance, considering coil-3, in which the EM pulse is optimally synchronised with $\sim 3.6 \mathrm{MeV}$ protons, simulations were carried out with a monochromatic proton source with input energies of $3,4,6$ and $8 \mathrm{MeV}$. As shown in Fig. 5(a), the energy gain for $3 \mathrm{MeV}$ protons is negative (i.e. undergoing deceleration) as the protons lag behind the strong electric field region inside the coil. The highest energy gain is attained by the protons with $4 \mathrm{MeV}$ input energy, which is closer to the synchronisation window of the coil $(\sim 3.6 \mathrm{MeV})$. As can be seen in the Fig. 5(a), a net energy gain of $2 \mathrm{MeV}$ is obtained using a $12 \mathrm{~mm}$ long coil, however, the energy gain profile tends to saturate part way through the coil. For higher input energies $(\sim 6 \mathrm{MeV})$, the energy gain is relatively small compared to the case of $4 \mathrm{MeV}$ input energy, and the gain profile saturates after travelling a much smaller distance $(\sim 3 \mathrm{~mm})$ inside the coil.

An insight to the sigmoidal behaviour of the energy gain profile shown in Fig. 5(a) for the case of $4 \mathrm{MeV}$ input energy can be obtained by considering, for instance, the section of the coil with the EM pulse as a uniformly charged ring 37. The charged ring therefore propagates along the coil axis with a velocity calculated from the position-time graph shown in Fig. 1(b). The longitudinal component of the electric field due to the charged ring along the coil axis is proportional to $\propto z\left(z^{2}+R^{2}\right)^{-3 / 2}$ as shown in Fig. 1 (c), which peaks at a distance of $\pm R / \sqrt{2}$ from the ring centre $(z=0)$. However, the transverse component of the electric field produced by the charged ring peaks at the centre of the ring. Therefore, a proton on the plane of the charged ring and travelling in synchronisation with the charged ring will experience a negligible longitudinal component of the electric field, while being strongly focused by the transverse component. Therefore, the $4 \mathrm{MeV}$ protons from the source, which travel in synchronisation with the EM pulse, are mainly focussed in the first few millimetres of the coil with minimal energy gain, until they advance relative to the EM pulse to the region of stronger longitudinal field, gaining energy at a higher rate.

Since the longitudinal field along the coil axis decreases after attaining its peak at $R / \sqrt{2}$, the rate of energy gain will eventually drop as the protons overrun the EM pulse significantly. This results in the saturation of energy gain seen in Fig. 5(a), for instance after $\sim 8 \mathrm{~mm}$ for $4 \mathrm{MeV}$ and $\sim 3 \mathrm{~mm}$ for $6 \mathrm{MeV}$ input energies. This problem can be 
Dynamics of guided post-acceleration of protons in a laser-driven travelling-field accelerator 8

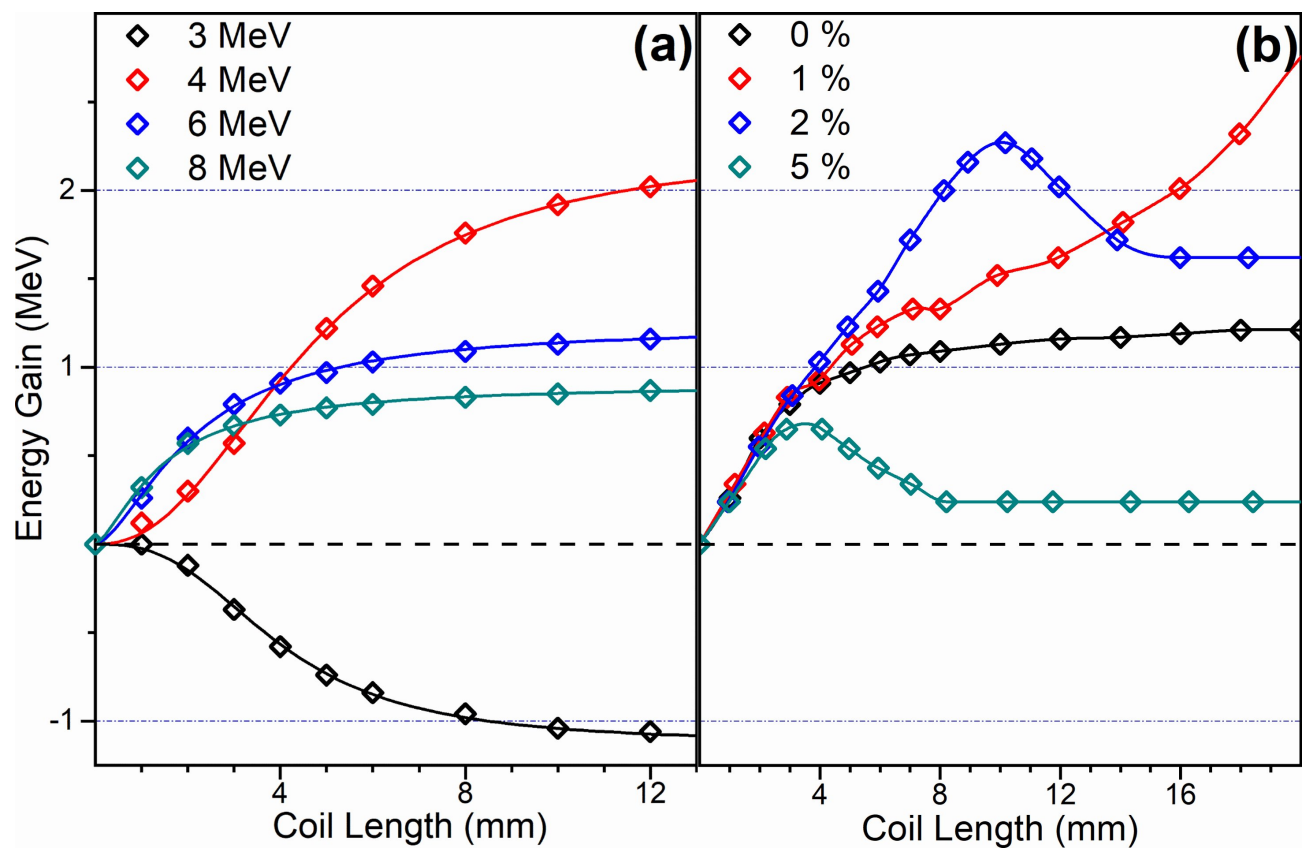

Figure 5. (a) Energy gain versus the length of the coil for the coil-3 case. The black, red, blue and green lines correspond to input energies of $2 \mathrm{MeV}, 4 \mathrm{MeV}$, $6 \mathrm{MeV}$ and $8 \mathrm{MeV}$ respectively. (b) Energy gain for $6 \mathrm{MeV}$ protons versus the length of the coil, for coils of $230 \mu \mathrm{m}$ initial pitch and constantly increasing the pitch by $0 \%$ (black), $1 \%$ (red), $2 \%$ (blue) and $5 \%$ (green).

mitigated by steadily increasing the pitch of the coil, which would allow a synchronous flow of the EM pulse with the transiting protons. Therefore, further simulations were carried out using uniformly varying-pitch coils, with the pitch increasing $1 \%, 2 \%$ and $5 \%$ of the initial pitch in each turn, as shown in Fig. 5 (b). The coil had an initial pitch of $230 \mu \mathrm{m}$ and $6 \mathrm{MeV}$ input energy was used for this study. As can be seen in Fig. 5(b), increasing the pitch by $1 \%$ the energy gain does not saturate even for a $20 \mathrm{~mm}$ long coil. However, if we increase the pitch by $2 \%$, protons start to decelerate after $10 \mathrm{~mm}$ of the coil. In this case the increase in pitch is therefore more than that is required to maintain a synchronisation between the accelerated protons and the EM pulse, eventually leading the protons falling behind the EM pulse into the trailing part of the field region, where the longitudinal field is directed towards the proton source. By increasing further the pitch increment to $2 \%$, the deceleration phase occurs much earlier in the coil, resulting in an almost negligible net energy gain.

This simple set of simulations indicates that not only the pitch variation has to be calculated precisely, but also one may need to vary the pitch non-uniformly along the coil length. A simple analytical model can be developed to design a suitable varyingpitch coil. The energy gain can be optimised if the protons stay always at a fixed distance (ideally, $R / \sqrt{2}$ ) in front of the peak of the EM pulse, i.e. both must travel with the same velocity along the axis of the coil. The velocity of protons of energy $E_{p}$ can be written as

$$
u_{p}=c \sqrt{1-\left(\frac{E_{p}}{m_{p} c^{2}}+1\right)^{-2}}
$$


Dynamics of guided post-acceleration of protons in a laser-driven travelling-field accelerator 9

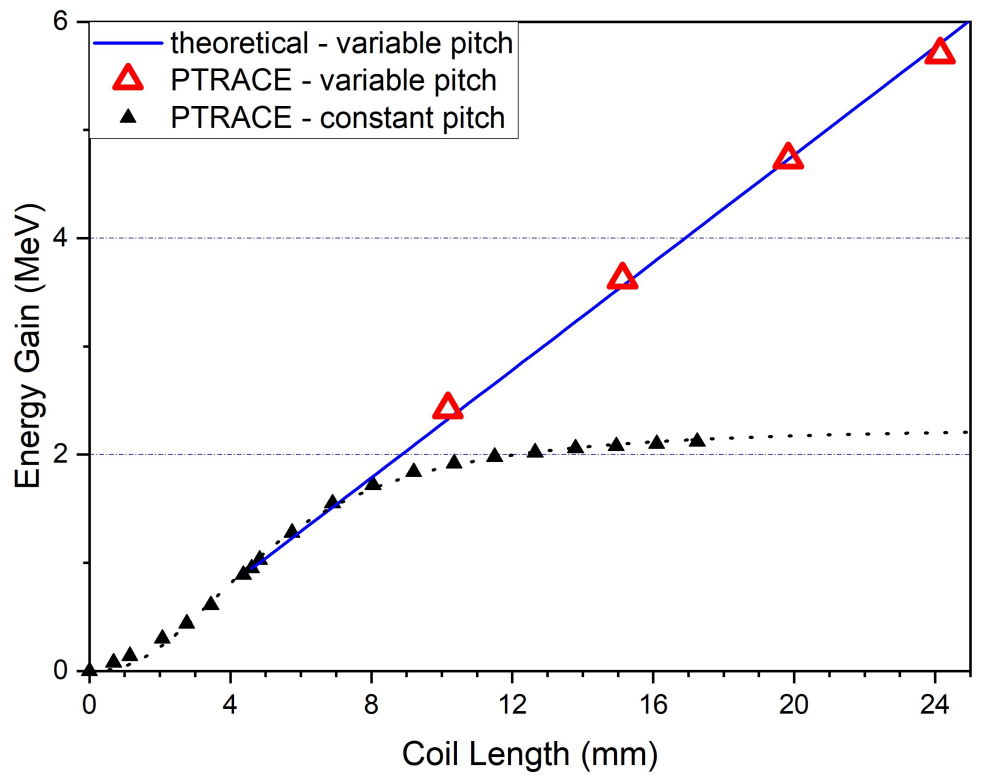

Figure 6. Energy gain versus coil length for $4 \mathrm{MeV}$ input protons are plotted for constant and varying pitch coils. The blue line corresponds to the energy gain for the latter case calculated analytically, while the red points are obtained by PTRACE simulations. For comparison, the black line shows the energy gain profile for the uniformly pitched coil-3 with $4 \mathrm{MeV}$ input energy, as shown in Fig. 5(a).

where $m_{p}$ is rest mass of protons. Considering that over one pitch length, $d$, the pulse travels a helical path of, $s=\sqrt{(2 \pi R)^{2}+d^{2}}$ with a velocity of $u_{c}=\beta c$ (where $\beta$ is found to be $0.96 \pm 0.4$ [3]), the velocity of the field region along the coil axis can be expressed as

$$
u_{c x}=\beta c\left(\frac{d}{s}\right)=\beta c\left[\left(\frac{2 \pi R}{d}\right)^{2}+1\right]^{-1 / 2} .
$$

Equating the two velocities and solving for the pitch gives

$$
d=2 \pi R \sqrt{\frac{\left(E_{p} m_{p}^{-1} c^{-2}+1\right)^{2}-1}{\left(\beta^{2}-1\right)\left(E_{p} m_{p}^{-1} c^{-2}+1\right)^{2}+1}} .
$$

Let us consider the $4 \mathrm{MeV}$ input protons, which produced the pencil beams with highest energy gain in a constant pitch coil, as shown in Fig. 4 and 5(a). As per Fig. 5(a), the energy gain profile takes a sigmoidal form for the first few millimetres of the coil length, followed by an energy gain at a rate $E_{g m} \sim 250 \mathrm{MeV} / \mathrm{m}$. After the first $4.6 \mathrm{~mm}$, the net energy gain was $E_{g}=\sim 1.1 \mathrm{MeV}$. Knowing the proton energy at a given position, $E_{p i}$, and the optimum pitch length for the next helical turn by using equation 3. one can estimate the output proton energy, $E_{p o}$, at the end of each turn by

$$
E_{p o}=E_{p i}+E_{g m} d .
$$

Following this procedure, a pitch table was constructed for the part of the coil after $z=4.6 \mathrm{~mm}$ in order to maintain energy gain at a rate $E_{g m}$ for an extended length 
of the coil. Although calculating coarsely over every pitch length gives reasonable estimates for the optimum pitches, it is worth to note that one should recalculate the energies for small fractions of a pitch in order to obtain a more accurate pitch table.

Fig. 6 shows the theoretically expected energy gain profile for a coil using the obtained pitch table, which agrees well with the PTRACE simulations (red points). The simulations were carried out for the same coil design and $4 \mathrm{MeV}$ input energy.

As can be seen, varying the pitch of the coil suitably can lead to a sustained energy gain over an extended length of the coil, which represents a step-forward optimising the energy gain by the helical coil targets. For instance, significantly stronger EM pulse is expected from the interaction of multi-petawatt lasers, which would lead producing acceleration gradients order of magnitude higher [37] than that demonstrated here $(\sim 0.25 \mathrm{MeV} / \mathrm{mm})$ using a university-scale laser system. A sustained post-acceleration at a rate of multi- $\mathrm{MeV} / \mathrm{mm}$ using suitably designed variable pitched coils can enable producing quasi-collimated, narrow-band proton beams of energies approaching therapeutic range.

\section{Conclusions}

In conclusions, we report on the characterisation of recently developed helical coil targets by deploying at $200 \mathrm{TW}$ laser system. The effect of the coil's length on chromatic guiding of proton beams was investigated. The experimental results show that the acceleration is suppressed for constant pitched coils after a certain length of the coil, which is due to de-phasing of the electric field region from the protons as the protons are accelerated while transiting through the coil.

In order to keep the accelerated protons synchronous with the EM pulse, a coil with increasing pitch would be warranted. With the help of a simple theoretical model and particle tracing simulations, a coil design keeping few-MeV protons synchronised with the EM pulse is presented. The concept of varying pitch coil can be, in principle, extended to high energy protons.

Authors acknowledge funding from EPSRC [EP/J002550/1 - Career Acceleration Fellowship held by S.K., EP/L002221/1, EP/K022415/1, EP/J500094/1, and $\mathrm{EP} / \mathrm{I} 029206 / 1]$, and Invest Northern Ireland (POC-329). This work is also supported by the project High Field Initiative (CZ.02.1.01/0.0/0.0/15_003/0000449) from the European Regional Development Fund. Authors also acknowledge A. Schiavi for the use of the particle tracing code, PTRACE.

[1] McKenna P et al 2006 "Demonstration of Fusion-Evaporation and Direct-Interaction Nuclear Reactions using High-Intensity Laser-Plasma-Accelerated Ion Beams" Phys. Rev. Lett. 91 075006

[2] Bulanov S V et al 2002 "Feasibility of Using Laser Ion Accelerators in Proton Therapy" Plasma Phys. Rep. 28 453-456

[3] Bulanov S V et al 2002 "Oncological hadrontherapy with laser ion accelerators" Phys. Lett. A 299 240-247

[4] Bulanov S V et al 2004 "Feasibility of Using Laser Ion Accelerators in Proton Therapy" AIP Conf. Proc. $\mathbf{7 4 0} 414$

[5] Hannachi F et al 2007 "Prospects for nuclear physics with lasers" Plasma Phys. Control. Fusion 49 B79

[6] Tabak M et al 1994 "Ignition and high gain with ultrapowerful lasers" Phys. Plasmas 11626

[7] Borghesi M et al 2002 "Electric field detection in laser-plasma interaction experiments via the proton imaging technique" Phys. Plasmas 9 2214-2220 
Dynamics of guided post-acceleration of protons in a laser-driven travelling-field accelerator 11

[8] Borghesi M et al 2003 "Measurement of highly transient electrical charging following high intensity laser-solid interaction" Appl. Phys. Lett. 82 1529-1531

[9] Quinn K et al 2009 "Observation of the transient charging of a laser-irradiated solid" Eur. Phys. J. D 55 293-297

[10] Quinn K et al 2009 "Laser-Driven Ultrafast Field Propagation on Solid Surfaces" Phys. Rev. Lett. 102194801

[11] Macchi A et al 2013 "Ion acceleration by superintense laser-plasma interaction" Rev. Mod. Phys. 85751

[12] Wagner F et al 2016 "Maximum Proton Energy above $85 \mathrm{MeV}$ from the Relativistic Interaction of Laser Pulses with Micrometer Thick $\mathrm{CH}_{2}$ Targets" Phys. Rev. Lett. 116205002

[13] Poole P L et al 2018 "Laser-driven ion acceleration via target normal sheath acceleration in the relativistic transparency regime" New J. Phys. 20013019

[14] Wang W P et al 2013 "Effects of nanosecond-scale prepulse on generation of high-energy protons in target normal sheath acceleration" Appl. Phys. Lett. 102224101

[15] Patel P K et al 2003 "Isochoric Heating of Solid-Density Matter with an Ultrafast Proton Beam" Phys. Rev. Lett. 91125004

[16] Ferre J et al 2019 "Enhanced target normal sheath acceleration using colliding laser pulses" Commun. Phys. 240

[17] Afshari M et al 2020 "Proton acceleration via the TNSA mechanism using a smoothed laser focus" AIP Adv. 10035023

[18] Pelka A et al 2010 "Ultrafast Melting of Carbon Induced by Intense Proton Beams" Phys. Rev. Lett. 105265701

[19] Dromey B et al 2016 "Picosecond metrology of laser-driven proton bursts" Nat. Comm. 710642

[20] Doria D et al 2012 "Biological cell irradiation at ultrahigh dose rate employing laser driven protons" AIP Conf. Proc. 1462135

[21] Nishiuchii M et al 2006 "The laser proton acceleration in the strong charge separation regime" Phys. Lett. A 357 339-344

[22] Busold S et al 2013 "Focusing and transport of high-intensity multi-MeV proton bunches from a compact laser-driven source" Phys. Rev. Spec. Top. - Ac. 16101302

[23] Schollmeier M et al 2008 "Controlled Transport and Focusing of Laser-Accelerated Protons with Miniature Magnetic Devices" Phys. Rev. Lett. 101055004

[24] Willi O et al 2007 "Laser triggered micro-lens for focusing and energy selection of MeV protons" Laser Part. Beams 25 71-77

[25] Borghesi M et al 2008 "Laser-Driven Proton Beams: Acceleration Mechanism, Beam Optimization, and Radiographic Applications" IEEE T. Plasma Sci. 36 1833-1842

[26] Toncan T et al 2006 "Ultrafast Laser-Driven Microlens to Focus and Energy-Select MegaElectron Volt Protons" Science $\mathbf{3 1 2} 410$

[27] Chen S N et al 2012 "Focusing dynamics of high-energy density, laser-driven ion beams" Phys. Rev. Lett. 108055001

[28] Kaymak V et al 2019 "Boosted acceleration of protons by tailored ultra-thin foil targets" Sci. Rep. 91

[29] Kar S et al 2008 "Dynamic Control of Laser-Produced Proton Beams" Phys. Rev. Lett. 100 105004

[30] Pfotenhauer S M et al 2010 "A cascaded laser acceleration scheme for the generation of spectrally controlled proton beams" New J. Phys. 10103009

[31] Schwoerer $\mathrm{H}$ et al 2006 "Laser-plasma acceleration of quasi-monoenergetic protons from microstructured targets" Nature Letters 439 445-448

[32] Offermann D T et al 2010 "Carbon ion beam focusing using laser irradiated, heated diamond hemispherical shells" J. Phys. Conf. Ser. 244022053

[33] Carrie M et al 2011 "Focusing of laser-accelerated proton beams using curved targets, and improved energy deposition" High Energy Density Phys. 7 353-360

[34] Qiao B et al 2013 "Dynamics of high-energy proton beam acceleration and focusing from hemisphere-cone targets by high-intensity lasers" Phys. Rev. E 87013108

[35] Bartal T et al 2011 "Focusing of short-pulse high-intensity laser-accelerated proton beams" Nat. Phys. 8 139-142

[36] Gaillard S A et al 2011 "Increased laser-accelerated proton energies via direct laser-light pressure acceleration of electrons in microcone targets" Phys. Plasmas 18056710

[37] Kar S et al 2016 "Guided post-acceleration of laser-driven ions by a miniature modular structure" Nat. Commun. 710792

[38] Willi O et al 2009 "Particle and x-ray generation by irradiation of gaseous and solid targets with a 100TW laser pulse" Plasma Phys. Control. Fusion 51124049 
Dynamics of guided post-acceleration of protons in a laser-driven travelling-field accelerator 12

[39] Cerchez M et al 2019 "ARCTURUS laser: a versatile high-contrast, high-power multi-beam laser system" High Power Laser Sci. Eng. 7 e37

[40] http://www.gafchromic.com/

[41] Schiavi A 2003 "PhD thesis" (Imperial College London) 